\title{
Liver cirrhosis in patients newly diagnosed with neurological phenotype of Wilson's disease
}

\author{
Adam Przybyłkowski, MD, PhDa \\ Grażyna Gromadzka MD, PhD ${ }^{a, b}$ \\ Grzegorz Chabik, MD \\ Agata Wierzchowska, MD \\ Tomasz Litwin, MD, PhD \\ Anna Członkowska, MD, PhDa,b
}

\begin{abstract}
${ }^{a}$ Department of Experimental and Clinical Pharmacology, Medical University of Warsaw, Warsaw, Poland

${ }^{\circ}$ Second Department of Neurology, Institute of Psychiatry and Neurology, Warsaw, Poland
\end{abstract}

Correspondence to: Adam Przybyłkowski

E-mail: aprzybylkowski@wum.edu.pl

\section{Summary}

Wilson's disease (WD) can manifest itself in different clinical forms, the neurological and hepatic ones being the most common. It is suggested that neurological signs and psychiatric symptoms develop secondary to liver involvement. The aim of this study was to characterize the liver disease in patients newly diagnosed with the neurological form of WD.

Treatment-naive patients diagnosed with WD were classified into three phenotypic groups: hepatic, neurological and pre-symptomatic. Liver involvement was ascertained through surrogate markers: abdominal ultrasound and laboratory parameters. In addition, study participants were screened for esophageal varices.

Of 53 consecutively diagnosed WD patients, 23 individuals $(43.4 \%)$ had a predominantly neurological presentation. In this group, cirrhosis was diagnosed in $11(47.8 \%)$ subjects. Esophageal varices were present in all of them. In every patient with neurological WD, there was at least one sign of hepatic disease on ultrasound examination, indicating universal presence of liver involvement. The prevalence of surrogate signs of cirrhosis was similar in patients with the neurological and in those with the hepatic phenotype.

KEY WORDS: esophageal varices, liver cirrhosis, Wilson's disease
Introduction

Wilson's disease (WD) is an autosomal recessive inborn error of metabolism which results in defective copper homeostasis and systemic copper toxicosis. The metabolic defect is due to impaired copper transport resulting from mutations in the $A T P 7 B$ gene encoding $P$-type adenosine triphosphatase (Tanzi et al., 1993). ATP7B is involved in excretion of copper from hepatocytes into the bile. The biallelic ATP7B mutation in WD patients is responsible for copper accumulation within the liver and other organs. This copper overload leads to organ damage and the clinical manifestations of WD (Ala et al., 2007). Three broad categories are recognized on the basis of the signs and symptoms: hepatic, neurological and pre-symptomatic (asymtopmatic). Furthermore, there is a wide clinical spectrum even within each of these phenotypic categories. For example, liver disease in patients classified as hepatic WD ranges from asymptomatic elevation of liver enzymes to acute hepatitis, occult cirrhosis and fulminant hepatic failure. Similarly, neurogical WD patients show a wide clinical spectrum of neurological manifestations, which range from behavioral symptoms to incapacitating movement disorder (Ala et al., 2007).

The natural history of WD is still not fully understood. It is not clear whether neurological signs and symptoms occur early in the course of the disease when liver involvement is mild (i.e., pre-cirrhotic) or whether they appear after the development of liver damage and cirrhosis. It has been suggested that neuropsychiatric symptoms in WD develop secondary to liver involvement (Harris et al., 2007). However, there is a paucity of information on the true prevalence and staging of liver disease among patients with neurological symptomatology. Correct staging of liver disease, especially the detection of advanced liver fibrosis or cirrhosis in patients with WD, is of therapeutic and prognostic importance. For example, the presence of cirrhosis alters the management of patients as regards monitoring for esophageal and gastric varices and hepatocellular carcinoma (D'Amico and Luca, 1997; Schuppan and Afdhal, 2008; Walshe et al., 2003). Histological examination of percutaneous biopsy specimens is the gold standard for assessment of fibrosis and cirrhosis; however, liver biopsy is an invasive procedure. Serum markers and ultrasonographic characteristics of liver parenchyma may be used as surrogate markers of cirrhosis (Schuppan and Afdhal, 2008). Ultrasonography (USG) is a non-invasive and inexpensive procedure for the diagnosis of parenchymal diseases of the liver. The sensitivity and specificity of USG in the detection of cirrhosis vary between authors, showing that this method 
is highly dependent on the examiner's experience. Nevertheless it is believed that USG can show liver cirrhosis with acceptable diagnostic accuracy (Aube et al., 2004). In this prospective study, we aimed to determine the extent of liver disease through surrogate laboratory markers and abdominal ultrasound in a consecutive series of newly diagnosed WD patients with the neurological phenotype, comparing the findings with those recorded in patients showing a predominantly hepatic presentation. In addition, we present the results of endoscopic screening for portal hypertension signs.

\section{Materials and methods}

\section{Patients}

A consecutive series of 53 adult patients diagnosed with WD at the Second Department of Neurology, Institute of Psychiatry and Neurology, Warsaw, Poland, and at the Department of Gastroenterology, Saint Ann's Hospital, Warsaw, Poland, were included in this study. Blood samples were obtained on admission to hospital. USG and endoscopy were performed during initial evaluation for WD. The study was approved by the local ethics committee and informed consent was obtained from all the patients. The study protocol conforms to the ethical guidelines of the 1975 Declaration of Helsinki as reflected in a priori approval by the institution's human research committee.

All patients met the WD diagnostic criteria of the $8^{\text {th }}$ International Meeting on Wilson disease and Menkes disease (Ferenci et al., 2003). No patient enrolled in the study had received any treatment for WD. The extent of the disease was ascertained on the basis of the presence of hepatic, neurological or psychiatric signs and symptoms.

The comprehensive evaluation allowed the patients to be classified into three phenotypic categories according to criteria proposed by Ferenci et al. (2003):

- patients with symptomatic liver disease without neurological and psychiatric symptoms were classified as having a hepatic presentation;

- patients in whom neurological and/or psychiatric symptoms were present at diagnosis were classified as neurological WD;

- patients without hepatic and neuropsychiatric symptoms (siblings of affected WD patients diagnosed through family screening) were classified as presymptomatic WD.

\section{Liver function tests and non-invasive markers of liver fibrosis/cirrhosis}

For the assessment of liver involvement, a battery of blood tests was performed in all the patients. Detoxification and excretory functions of the liver were assessed determining the activity of: alanine aminotransferase (ALT), asparagine aminotransferase (AST) and gamma-glutamyl transpeptidase (GGTP). In addition, bilirubin concentration was measured. Biosynthetic function of the liver was ascertained by measuring serum albumin concentration and international normalized ratio (INR). Thrombocytopenia resulting from sequestration of platelets in congested spleen is frequently present in patients with chronic liver disease; therefore, platelet count was measured in all the patients. All tests were performed using standard laboratory methods.

The following non-invasive markers of liver fibrosis were calculated: i) AST/ALT ratio (AAR); ii) AST to platelet ratio index (APRI). The APRI is a routine biochemical and hematological test created by Wai et al. (2003) to evaluate the presence of liver cirrhosis. An AAR cutoff value of 1.0 was set for predicting advanced fibrosis (Manning and Afdhal, 2008), while the APRI threshold value for severe fibrosis was set at 1.0.

\section{Abdominal ultrasound examination}

Ultrasound examination of the liver was performed according to a uniform protocol using a Philips HD11XE (Philips Healthcare, Best, The Netherlands), equipped with $2-5 \mathrm{MHz}$ broadband curved array transducer. The following seven variables for advanced liver fibrosis/cirrhosis were assessed: nodular liver surface, inhomogeneity of liver parenchyma, presence of collateral circulation, splenomegaly, ascites, portal vein diameter and mean velocity of the flow in the portal vein (Aube et al., 1999). The surface nodularity variable was considered positive if, instead of a straight and regular hyperechoic line, the liver surface appeared as a dotted or irregular line. If the liver parenchyma was not homogeneous but showed areas with different echogenicity, parenchymal inhomogeneity was deemed to be present. Collateral circulation was defined as umbilical vein diameter $>3 \mathrm{~mm}$ or a left gastric vein $>5 \mathrm{~mm}$ or any abnormal vein such as a spleno-renal shunt. Spleen size was assessed by the longitudinal section and the spleen was considered enlarged when this was $>13 \mathrm{~cm}$ (Frank et al., 1986). Ascites was recorded when present. Portal vein diameter was considered abnormal when it was $>13 \mathrm{~mm}$ (Goyal et al., 2009). For portal vein assessment, a mean flow velocity cut-off value of $15 \mathrm{~cm} / \mathrm{s}$ was used (Goyal et al., 2009). For the diagnosis of cirrhosis, the following ultrasonographic criteria were arbitrarily established: presence of nodular surface accompanied by at least two other signs of liver fibrosis.

\section{Endoscopy}

Upper gastrointestinal tract endoscopy was performed by two experienced endoscopists. The patients were screened for the presence of esophageal varices, portal-hypertensive gastropathy and gastric varices. Esophageal varix stage was defined according to the Italian Liver Cirrhosis Project (ILCP) criteria (Merkel et al., 2000). ILCP group staging of variceal size is a function of the percentage of the esophageal lumen occupied by the largest varix: grade 1 - varix occupies $<25 \%$ of the esophageal lumen, grade 2 - varix occupies 25 $50 \%$ of the esophageal lumen, and grade 3 - varix occupies $>50 \%$ of the esophageal lumen. 


\section{Statistical analysis}

The data were analyzed using STATISTICA 10.0 (StatSoft, Cracow, Poland). The normality of analyzed variables was determined using the KolmogorovSmirnov and Lilliefors tests. Normally distributed variables were presented as a mean and standard deviation. Variables that were not normally distributed in the analyzed population were presented as median and interquartile range. Student's t-test and the MannWhitney $U$ test were used for further analysis of normally/not-normally distributed variables, respectively. Categorical variables were compared between groups by Fisher's exact test. Relationships between variables were examined using the Spearman rank correlation coefficient. The significance level was set at 0.05 .

\section{Results}

\section{Patient characteristics}

Fifty-three consecutively diagnosed patients were phenotyped as hepatic or neurological WD. The baseline characteristics of the patients are presented in table I. The symptoms and signs suggestive of WD were neuropsychiatric in 23 cases $(43.4 \%)$ and hepatic in 19 cases $(35.8 \%)$; the other 11 individuals were evaluated for WD after diagnosis of the disease in a sibling $(20.7 \%)$. The study group comprised 25 (47.2\%) women and 28 (52.8\%) men (Table I). The mean age at diagnosis of WD was significantly later in the neurological phenotype group compared with the hepatic group: 35.3 years (range 18-50) vs 26.3 years (range 18-57; $\mathrm{p}<0.02$ ). The mean age at diagnosis of asymptomatic patients diagnosed through family screening was 27.8 years (range 20-44). The mean symptom duration before diagnosis did not differ between the groups.

On physical examination, hepatic signs were present in $43.5 \%$ of the neurological group patients, with peripheral edema being the most common sign (Table I). Jaundice was present in three neurological patients, while one subject presented fulminant liver failure (Table I). Tremor was the most frequent neurological abnormality, while 11 patients complained of psychiatric symptoms. Kayser-Fleischer (KF) ring was detected in all 23 patients in the neurological group compared with 11 patients in the hepatic phenotype group $(57.9 \%$; $p<0.0003)$. Four pre-symptomatic indi-

Table I - Baseline and clinical characteristics of the Wilson's disease patients participating in the study.

\begin{tabular}{|c|c|c|c|}
\hline Clinical phenotype & $\begin{array}{l}\text { Neurological } \\
(n=23)\end{array}$ & $\begin{array}{l}\text { Hepatic } \\
(n=19)\end{array}$ & $\begin{array}{l}\text { Pre-symptomatic } \\
(n=11)\end{array}$ \\
\hline Age at diagnosis (years), mean $\pm \mathrm{SD}$ & $35.3 \pm 11.2^{a}$ & $26 \pm 6.7$ & $27.8 \pm 8.0$ \\
\hline \multicolumn{4}{|l|}{ Sex, $n(\%)$} \\
\hline - female & $10(43.5)$ & $8(42.1)$ & $7(63.6)$ \\
\hline - male & $13(56.5)$ & $11(57.9)$ & $4(36.4)$ \\
\hline $\begin{array}{l}\text { Duration of symptoms in years, } \\
\text { median (IQR) }\end{array}$ & $1.0(2.0)$ & $1.0(1.0)$ & 0 \\
\hline Hepatic signs, $n(\%)$ : & $10(43.5)$ & $19(100.0)$ & - \\
\hline - hepatomegaly, $n(\%)$ & $6(26.1)$ & $8(42.1)$ & - \\
\hline - jaundice, $n(\%)$ & $3(13.0)$ & $4(21.1)$ & - \\
\hline - ascites, $n(\%)$ & $2(8.7)$ & $3(15.8)$ & - \\
\hline - peripheral edema, $n(\%)$ & $9(39.1)$ & $10(52.6)$ & - \\
\hline \multicolumn{4}{|l|}{ Neurological signs: } \\
\hline - tremor, $n(\%)$ & $15(65.2)$ & - & - \\
\hline - rigidity, $n(\%)$ & $5(21.7)$ & - & - \\
\hline - dystonia, $n(\%)$ & $5(21.7)$ & - & - \\
\hline - ataxia, $n(\%)$ & $5(21.7)$ & - & - \\
\hline Psychiatric symptoms, $n(\%)$ & $11(47.8)$ & - & - \\
\hline Kayser-Fleischer ring; $n(\%)$ & $23(100.0)^{a}$ & $11(57.9)$ & $4(36.4)$ \\
\hline $\begin{array}{l}\text { Serum ceruloplasmin in } \mathrm{mg} / \mathrm{dL} \\
\text { (normal: } 25-45 \text { ); median (IQR) }\end{array}$ & $15.1(28.5)$ & $16.7(37.5)$ & $17.9(24.4)$ \\
\hline $\begin{array}{l}\text { Serum copper in } \mu \mathrm{g} / \mathrm{dL} \\
\text { (normal: } 70-140 \text { ); median (IQR) }\end{array}$ & $42.7(39.2)$ & $46.0(38.9)$ & $63.9(28.9)$ \\
\hline $\begin{array}{l}\text { Copper excretion in urine within } 24 \mathrm{~h} \text { in } \\
\mu \mathrm{g} / 24 \mathrm{~h} \text { (normal: } 0-50) \text {; median (IQR) }\end{array}$ & $141.0(297.0)$ & $219.5(141.0)$ & $83.0(306.0)$ \\
\hline
\end{tabular}

Abbreviations: $\mathrm{SD}=$ standard deviation; $\mathrm{IQR}=$ interquartile range.

a $\mathrm{p}<0.05$ for the comparison with the hepatic group. Student's t-test or Mann-Whitney $U$ test for normally/not normally distributed variables, respectively. Categorical variables were compared between groups by Fisher's exact test. 
viduals (16.4\%) diagnosed through family screening also had KF rings, a finding that underscores the insidiously progressive nature of WD. Table II shows the results of mutation analysis of the $A T P 7 B$ gene. The $\mathrm{H} 1069 \mathrm{Q}$ mutation was the most common.

\section{Non-invasive markers of liver fibrosis/cirrhosis}

A significantly lower prevalence of abnormal liver function tests was found among the patients exhibiting the neurological phenotype compared with the hepatic phenotype; ALT and AST were elevated in $30.4 \%$ of the neurological WD patients compared with $63.2 \%$ of the hepatic patients $(p<0.03)$; GGTP was elevated in $17.4 \%$ vs $73.7 \%$, respectively $(p<0.0003)$. Moreover, the groups differed in absolute values of laboratory tests (Table III). Serum bilirubin, albumin, INR and platelet count values did not differ significantly between the hepatic phenotype group and the neurological group. An AAR $>1$, positive for fibrosis, was found in $16(69.6 \%)$ neurological WD patients and in $10(52.6 \%)$ patients with the hepatic phenotype. Six asymptomatic subjects were also positive for AAR. APRI>1 was present in eight $(34.8 \%)$ neurological patients and nine $(47.4 \%)$ hepatic patients, but none from the family screening group.

\section{Abdominal ultrasound examination}

Irregular liver surface and parenchymal inhomogeneity were present more frequently in the neurological WD patients than in the hepatic WD patients (Table IV). Irregular surface was not observed in any patient from the family screening group, but parenchymal inhomogeneity was observed in three $(27.3 \%)$ of them. Collateral circulation indicating underlying portal hypertension was present in a minority of WD patients (two patients with the neurological and two with the hepatic phenotype). Splenomegaly was detected in seven (30.4\%) neurological WD patients and in seven $(36.8 \%)$ hepatic WD patients. Interestingly, splenomegaly was present in one asymptomatic patient diagnosed through family screening. Free fluid in the peritoneal cavity was present in three (13.0\%) neurological WD patients compared with five (26.3\%) patients exhibiting the hepatic phenotype. The frequency of portal vein dilatation and the prevalence of decreased portal flow were similar in patients with neurological and hepatic presentation, but these signs were rare in siblings and relatives of index cases. Ultrasonographic criteria for liver cirrhosis were fulfilled by $11(47.8 \%)$ neurological patients and by seven $(36.8 \%)$ hepatic patients. One patient diagnosed asymptomatically through family screening also exhibited ultrasonographic signs of advanced liver disease. In every patient with neurological WD, there was at least one ultrasonographic sign, indicating universal presence of liver involvement. The number of ultrasonographic signs correlated significantly with APRI $(\rho=0.5 ; \quad p<0.05)$ and with presence of esophageal varices $(\rho=0.3 ; p<0.05)$. No significant correlation was found between the AAR and the number of ultrasonographic signs.

\section{Endoscopy}

None of the studied WD patients suffered from variceal bleeding. However, grade 1-2 esophageal varices were present in $47.8 \%$ of patients with the neurological phenotype (Table V). In hepatic WD, grade 1-3 esophageal varices were detected in $36.9 \%$ of patients. Esophageal varices were present in one asymptomatic patient diagnosed through family screening (Table V). Neither portalhypertensive gastropathy nor gastric varices were detect-

Table II - Distribution of mutations of the ATP7B gene.

\begin{tabular}{lllll}
\hline Mutation & Exon & Nucleotide change & $n$ of allelles & Frequency (\%) \\
\hline p.H1069Q & 14 & c.3207C>A & 69 & 65.1 \\
p.Q1351X & 20 & c.4051C>T & 5 & 4.7 \\
p.A1135fs & 15 & c.3402delC & 3 & 2.8 \\
p.G1341D & 20 & c.4022G>A & 2 & 1.9 \\
p.P1273L & 18 & c.3818C>T & 2 & 1.9 \\
p.V1217_L1218del & 17 & c.3649_3654del6 & 2 & 1.9 \\
p.IVS16-2A>G & 17 & c.3557-2A>G & 2 & 1.9 \\
p.G1186C & 16 & c.3556G>T & 2 & 1.9 \\
p.G1158fs & 16 & c.3472_3482del & 2 & 1.9 \\
p.W1353S & 20 & c.4058G>C & 1 & 0.9 \\
p.I1148T & 16 & c.3443T>C & 1 & 0.9 \\
p.R969fs & 13 & c.2905delC & 1 & 0.9 \\
p.Q544X & 4 & c.1630C>T & 1 & 0.9 \\
p.A1135T & 15 & c.3403G>A & 1 & 0.9
\end{tabular}

The data do not total $100 \%$ as pathogenic mutations were not always identified in both alleles in all 53 patients. 
Table III - Liver function tests and indirect markers of liver fibrosis in patients with Wilson's disease.

\begin{tabular}{llll}
\hline Clinical phenotype & $\begin{array}{l}\text { Neurological } \\
(n=23)\end{array}$ & $\begin{array}{l}\text { Hepatic } \\
(n=19)\end{array}$ & $\begin{array}{l}\text { Pre-symptomatic } \\
(n=11)\end{array}$ \\
\hline ALT, IU/L (normal: 0-38), median (IQR) & $23.0(19.0)^{\mathrm{a}}$ & $53.0(51.0)$ & $29.4(29.0)^{\mathrm{a}}$ \\
Patients with elevated ALT, $n$ (\%) & $7(30.4)^{\mathrm{a}}$ & $12(63.2)$ & $3(27.3)$ \\
AST, IU/L (normal: 0-40), median (IQR) & $27.0(23.4)^{\mathrm{a}}$ & $46.0(24.0)$ & $30.0(15.0)^{\mathrm{a}}$ \\
Patients with elevated AST, $n$ (\%) & $7(30.4)^{\mathrm{a}}$ & $12(63.2)$ & $1(9.1)$ \\
GGTP (normal: 8.0-54.0), median (IQR) & $35.9(22.2)^{\mathrm{a}}$ & $106.0(86.4)$ & $42.0(48.2)^{\mathrm{a}}$ \\
Patients with elevated GGTP, $n$ (\%) & $4(17.4)^{\mathrm{a}}$ & $14(73.7)$ & $3(27.3)$ \\
Bilirubin in mg/dL (normal: 0.2-1.2), median (IQR) & $1.1(0.3)$ & $1.3(1.0)$ & $1.1(0.2)$ \\
Patients with elevated bilirubin, $n$ (\%) & $8(34.8)$ & $10(52.6)$ & 0 \\
Serum albumin in g/dL (normal: 3.5-5.3), mean \pm SD & $3.7 \pm 1.0$ & $3.8 \pm 1.7$ & $3.9 \pm 0.8$ \\
Patients with decreased serum albumin, $n(\%)$ & $9(39.1)$ & $8(42.1)$ & $1(9.1)$ \\
INR ratio (normal 0.8-1.2), mean \pm SD & $1.2 \pm 0.2$ & $1.3 \pm 0.6$ & $1.3 \pm 0.2$ \\
Patients with elevated INR, $n$ (\%) & $15(65.2)$ & $9(47.4)$ & 0 \\
Platelet count x10/L (normal: 140-440), median (IQR) & $125.0(97.0)$ & $120.0(128.0)$ & $231.0(84.0)^{\mathrm{a}, \mathrm{b}}$ \\
Patients with decreased platelet count, $n(\%)$ & $12(52.2)$ & $12(63.2)$ & 0 \\
AAR >1, $n$ (\%) & $16(69.6)$ & $10(52.6)$ & $6(54.5)$ \\
APRI > 1, $n$ (\%) & $8(34.8)$ & $9(47.4)$ & 0 \\
\hline
\end{tabular}

Abbreviations: $\mathrm{SD}=$ standard deviation; IQR=interquartile range; $\mathrm{AST}=$ asparagine aminotransferase; $\mathrm{ALT}=$ alanine aminotransferase; $\mathrm{GGTP}=\mathrm{gamma}$ glutamyl transpeptidase; INR=international normalized ratio; AAR=asparagine aminotransferase/alanine aminotransferase ratio; APRI= AST to platelet ratio index.

${ }^{a} \mathrm{p}<0.05$ for the comparison with the hepatic group; ${ }^{b} \mathrm{p}<0.05$ for the comparison with the neurological group. Student's t-test or Mann-Whitney $U$ test for normally/not normally distributed variables, respectively. Categorical variables were compared between groups by Fisher's exact test.

Table IV - Prevalence of ultrasonographic signs of liver cirrhosis in patients with Wilson's disease.

\begin{tabular}{llll}
\hline Clinical phenotype & $\begin{array}{l}\text { Neurological } \\
(n=23)\end{array}$ & $\begin{array}{l}\text { Hepatic } \\
(n=19)\end{array}$ & $\begin{array}{l}\text { Pre-symptomatic } \\
(n=11)\end{array}$ \\
\hline Irregular surface, $n(\%)$ & $15(65.2)^{\mathrm{a}}$ & $7(36.8)$ & 0 \\
Parenchymal inhomogeneity, $n(\%)$ & $21(91.3)^{\mathrm{a}}$ & $10(52.6)$ & $3(27.3)$ \\
Collateral circulation, $n(\%)$ & $2(8.7)$ & $2(10.5)$ & 0 \\
Free peritoneal fluid, $n(\%)$ & $3(13.0)$ & $5(26.3)$ & $1(9.1)$ \\
Enlargement of the spleen, $n(\%)$ & $7(30.4)$ & $7(36.8)$ & $1(9.1)$ \\
Dilated portal vein, $n(\%)$ & $11(47.8)$ & $11(57.9)$ & $1(9.1)$ \\
Decreased portal vein flow, $n(\%)$ & $8(34.8)$ & $9(47.4)$ & $2(18.2)$ \\
\hline
\end{tabular}

a $p<0.05$ for the comparison with the hepatic group; categorical variables were compared between the hepatic and the neurological group by Fisher's exact test.

Table V - Prevalence of esophageal varices.

\begin{tabular}{llll}
\hline Clinical phenotype & $\begin{array}{l}\text { Neurological } \\
(n=23)\end{array}$ & $\begin{array}{l}\text { Hepatic } \\
(n=19)\end{array}$ & $\begin{array}{l}\text { Pre-symptomatic } \\
(n=11)\end{array}$ \\
\hline Varices grade $1 ; n(\%)$ & $8(34.8)$ & $4(21.1)$ & $1(9.1)$ \\
Varices grade $2 ; n(\%)$ & $3(13.0)$ & $2(10.5)$ & 0 \\
Varices grade 3; $n(\%)$ & 0 & $1(5.3)$ & 0 \\
\hline
\end{tabular}

Italian Liver Cirrhosis Project (ILCP) criteria were applied to stage esophageal varices. 
ed in any patient. Presence of esophageal varices correlated significantly with number of ultrasonographic signs (as above). Correlations with single ultrasonographic signs were significant for liver homogeneity: $\rho=0.4$, $p<0.05$, collateral circulation: $\rho=0.4, p<0.05$, nodular surface: $\rho=0.3, p<0.05$, and dilated portal vein: $\rho=0.3$, $p<0.05$. A significant correlation was also found between esophageal varices and APRI 1.0: $\rho=0.4, p<0.05$.

\section{Discussion}

Although WD is a single gene disorder, it is highly heterogeneous in its manifestations. The basis of this phenotypic variation is unclear as of yet. Thus when a patient is classified as having neurological WD with no clinical evidence of liver disease, it is not known whether the patient harbors minimal liver involvement or, at the other extreme, advanced liver disease that is not revealed by standard criteria (i.e., liver function tests, liver synthetic function or clinical signs of portal hypertension). In this series of consecutively evaluated WD patients, a high prevalence of surrogate signs of advanced liver disease was found among all the patients with symptomatic disease. It is important to note that patients with neurological WD and no overt clinical signs of liver disease had a high prevalence of surrogate markers of cirrhosis. It is suggested that neurological symptoms in Wilsonian patients typically develop secondary to liver involvement (Harris et al., 2007). However, the true prevalence and staging of liver disease among patients with neurological symptomatology are not yet clearly defined. In one autopsy study, hepatitis and micro-/macronodular cirrhosis were observed in all patients with neurological presentation of the disease (Meenakshi-Sundaram et al., 2008). In another cohort of 30 patients, cirrhosis was also diagnosed in all the patients presenting neurological symptoms; however, this group comprised only three subjects (Gow et al., 2000).

Detection of cirrhosis is crucial for treatment planning (qualification for liver transplantation) and from the perspective of surveillance for cirrhosis complications such as portal hypertension. Analysis of deaths in WD revealed that liver failure and variceal bleeding are common causes (Walshe, 2007; Czlonkowska et al., 2005). USG-based diagnosis of liver cirrhosis can be based on hepatic and extra-hepatic signs, and the accuracy of USG varies across different studies also because of the different parameters examined (Aube et al., 1999). There is no approved score for ultrasonographic diagnosis of cirrhosis, thus in common practice the diagnosis is made at the examining physician's discretion (Bonekamp et al., 2009). In the present study, it was decided on the basis of the authors' experience that the presence of at least three ultrasonographic signs (one of which must be nodular surface of the liver) is reliable for liver cirrhosis diagnosis. Applying this criterion, liver cirrhosis was observed in 11 of the 23 $(47.8 \%)$ neurological patients. All of them had abnormal liver function tests and in all of them esophageal varices were detected. Interestingly, among 11 rela- tives who had never sought medical advice for hepatic or neurological problems, subtle liver involvement signs (and even esophageal varices in one case) were present. This observation strongly supports the idea that the family members of newly diagnosed WD patients should be screened as soon as possible and immediately started on anticopper therapy if the diagnosis of WD is confirmed in them.

At present, liver biopsy is still the reference standard for the assessment and staging of liver disease. Therefore, an obvious limitation of the present study is the lack of verification of the examined surrogate markers of liver cirrhosis with histological assessment of the liver parenchyma. In this study, liver biopsy was not performed routinely in all the WD patients. However biopsy confirmation of cirrhosis is not mandatory if clear signs of cirrhosis (ascites, coagulopathy, shrunken nodular-appearing liver on imaging studies) are present (Schuppan and Afdhal, 2008). Therefore, in some WD cases, conventional ultrasound examination in combination with endoscopy and laboratory test results may be sufficient to diagnose liver cirrhosis. In conclusion, using laboratory markers, ultrasonographic signs or endoscopy, cirrhosis was diagnosed in $47.8 \%$ of newly diagnosed patients with the neurological form of WD. Some of them had liver dysfunction signs in their medical history, but had not been further evaluated. In every patient with neurological WD, there was at least one ultrasonographic sign of advanced liver disease. These results indicate the need for detailed assessment of the liver in all newly diagnosed patients with neurological presentation of WD even if they do not show obvious hepatic signs and symptoms. Careful observation of liver function in these cases is essential in order to prevent severe complications (e.g. variceal bleeding) and for treatment strategy.

Acknowledgments: This study was supported by The National Science Centre (grant no. NN402250534).

\section{References}

Ala A, Walker AP, Ashkan K, et al (2007). Wilson's disease. Lancet 369: 397-408.

Aube C, Oberti F, Korali N, et al (1999). Ultrasonographic diagnosis of hepatic fibrosis or cirrhosis. J Hepatol 30: 472478.

Aube C, Winkfield B, Oberti F, et al (2004). New Doppler ultrasound signs improve the non-invasive diagnosis of cirrhosis or severe liver fibrosis. Eur J Gastroenterol Hepatol 16: 743-751.

Bonekamp S, Kamel I, Solga S, et al (2009). Can imaging modalities diagnose and stage hepatic fibrosis and cirrhosis accurately? J Hepatol 50: 17-35.

Członkowska A, Tarnacka B, Litwin T, et al (2005). Wilson's disease-cause of mortality in 164 patients during 1992-2003 observation period. J Neurol 252: 698-703.

D'Amico G, Luca A (1997). Natural history. Clinical-haemodynamic correlations. Prediction of the risk of bleeding. Baillieres Clin Gastroenterol 11: 243-256.

Ferenci P, Caca K, Loudianos G, et al (2003). Diagnosis and 
phenotypic classification of Wilson disease. Liver Int 23: 139-142.

Frank K, Linhart P, Kortsik C, et al (1986). [Sonographic determination of spleen size: normal dimensions in adults with a healthy spleen]. Ultraschall Med 7: 134-137.

Gow PJ, Smallwood RA, Angus PW, et al (2000). Diagnosis of Wilson's disease: an experience over three decades. Gut 46: 415-419.

Goyal N, Jain N, Rachapalli V, et al (2009). Non-invasive evaluation of liver cirrhosis using ultrasound. Clin Radiol 64: 1056-1066.

Harris S, Naina HV, Siddique S (2007). Wilson's disease. Lancet 369: 902-903.

Manning DS, Afdhal NH (2008) Diagnosis and quantitation of fibrosis. Gastroenterology 134: 1670-1681.

Meenakshi-Sundaram S, Mahadevan A, Taly AB, et al (2008). Wilson's disease: a clinico-neuropathological autopsy study. J Clin Neurosci 15: 409-417.

Merkel C, Zoli M, Siringo S, et al (2000). Prognostic indicators of risk for first variceal bleeding in cirrhosis: a multicenter study in 711 patients to validate and improve the North Italian Endoscopic Club (NIEC) index. Am J Gastroenterol 95: 2915-2920.

Schuppan D, Afdhal NH (2008). Liver cirrhosis. Lancet 371: 838-851.

Tanzi RE, Petrukhin K, Chernov I, et al. (1993). The Wilson disease gene is a copper transporting ATPase with homology to the Menkes disease gene. Nat Genet 5 : 344-350.

Wai CT, Greenson JK, Fontana RJ et al (2003). A simple noninvasive index can predict both significant fibrosis and cirrhosis in patients with chronic hepatitis C. Hepatology 38 : 518-526.

Walshe JM (2007). Cause of death in Wilson disease. Mov Disord 22:2216-2220.

Walshe JM, Waldenström E, Sams V, et al (2003). Abdominal malignancies in patients with Wilson's disease. QJM 96: 657-662. 